sondere wenn und weil dadurch andere in ihren Rechten betroffen werden. Dazu lässt sich nicht nur auf das Zitat von Immanuel Kant (1724 - 1804) verweisen: „Die Freiheit des Einzelnen endet dort, wo die Freiheit des Anderen beginnt." Denn in dem vorgenannten Szenario stören NichtBerechtigte (d.h. die gewerblichen Veranstalter und deren Fahrgäste) Berechtigte (d.h. „Rechtsinhaber“), die in einer allein privatrechtlichen Rechtsordnung sogar auf gänzliche Unterlassung der Störung in ihren Rechten klagen könnten.

Hier ist es deshalb die Aufgabe der Landesgesetzgeber, ggf. Gewässerstrecken zu bestimmen, auf denen mit Floß und Raft und Kanu „Party gemacht“ werden kann, um Regionen touristisch aufzuwerten. Dann sollte er aber auch die dafür benötigte Infrastruktur schaffen und zugleich verhindern, dass Konflikte mit Natur und Landschaft, Anwohnern und anderen Rechtsinhabern (wie hier die Angler und Angelvereine) auftreten.

Bis dahin gilt es aber für die zuständigen Wasserbehörden, unter Beachtung und Anwendung des geltenden Rechts einen fairen Interessenausgleich zu schaffen. Der vorliegende Beitrag könnte dabei helfen, diesen Interessenausgleich auf eine gesicherte rechtliche Basis zu stellen.

\title{
https://doi.org/10.1007/s10357-020-3640-2 \\ Aktuelle FFH-Judikatur zu erheblichen Beeinträchtigungen und FFH-VP
}

\section{Prof. Dr. Walter Frenz}

\begin{abstract}
CC Der/die Autor(en) 2020
Der eigentlichen FFH-Verträglichkeitsprüfung vorgelagert ist die Frage, ob erhebliche Beeinträchtigungen eines Habitatschutzgebietes vorliegen können. Diese Vorprüfung wurde konzeptionell verfeinert und im Hinblick auf die Methode der Critical Loads konkretisiert. Im Bereich der Verträglichkeitsprüfung geht es vor allem um die Bewältigung von Unsicherheiten.
\end{abstract}

\section{Zweistufige Prüfung nach dem EuGH}

Im Urteil Doel bekräftigte der EuGH seine Konzeption der zweiphasigen Prüfung, wie sie in Art. 6 Abs. 3 FFH-RL vorgezeichnet ist. Zunächst geht es nach Satz 1 um die Prüfung der Verträglichkeit eines Plans oder eines Projektes mit einem geschützten Gebiet, was voraussetzt, dass dieses Gebiet wahrscheinlich erheblich beeinträchtigt wird. Sodann ist nach der in Art. 6 Abs. 3 Satz 2 FFH-RL vorgesehenen Phase zu prüfen, ob das betreffende Gebiet als solches nicht wesentlich beeinträchtigt wird. ${ }^{1}$ Dafür sind nach den besten einschlägigen wissenschaftlichen Erkenntnissen sämtliche Gesichtspunkte des Plans oder Projekts zu ermitteln, die für sich oder i. V.m. anderen Plänen oder Projekten die für dieses Gebiet festgelegten Erhaltungsziele beeinträchtigen können. Dies muss ausgeschlossen sein. Die Behörden müssen Gewissheit darüber erlangt haben, dass aus wissenschaftlicher Sicht kein vernünftiger Zweifel daran besteht, dass es keine nachteiligen Auswirkungen auf das Gebiet als solches gibt. ${ }^{2}$ Dadurch wurde die Umkehr der Beweislast betont.

\section{Vorprüfung der erheblichen Beeinträchtigung}

Indem der EuGH für die Durchführung der Verträglichkeitsprüfung voraussetzt, dass das betroffene Gebiet wahrscheinlich erheblich beeinträchtigt wird, ist diese Frage vorher zu prüfen. Diese Voraussetzung muss mithin gegeben sein und ist eine Vorstufe, über die erst in die FFH-Verträglichkeitsprüfung eingetreten werden kann. Nur wenn eine wahrscheinliche erhebliche Beeinträchtigung vorliegt, ist also die Gebietsverträglichkeit zu prüfen, Die Frage einer erheblichen Beeinträchtigung bedarf daher einer Vorprüfung. ${ }^{3}$ Diese bildet die Grundlage für eine tiefere, in

Prof. Dr. Walter Frenz,

Lehrstuhl für Berg-, Umwelt- und Europarecht,

Rheinisch-Westfälische Technische Hochschule Aachen,

Aachen, Deutschland der maßgeblichen Ausrichtung auf die Erhaltungsziele des fraglichen Gebietes aber gleichgelagerte Untersuchung im Rahmen der FFH-Verträglichkeitsprüfung, ${ }^{4}$ welche die Möglichkeit einer erheblichen Beeinträchtigung ohne vernünftigen Zweifel ausschließen muss, damit ein Projekt ohne Abweichungsentscheidung nach $\$ 34$ Abs. 3 BNatSchG zugelassen werden kann.

Die darauf bezogene Vorprüfung muss nicht formalisiert durchgeführt werden; vielmehr geht es nur um die Feststellung, dass die Voraussetzungen nicht vorliegen, unter denen eine Verträglichkeitsprüfung geboten ist, weil eine Beeinträchtigung der Erhaltungsziele des Gebietes ohne vertiefte Prüfung ausgeschlossen werden kann. ${ }^{5}$ Bei dieser Vorprüfung erfolgt mithin eine ,überschlägige“ Beurteilung, „ohne die eigentliche Verträglichkeitsprüfung vorwegzunehmen". ${ }^{6}$ Sie ist dann unter Umständen nicht notwendig, der Verzicht auf sie kein Rechtsfehler. ${ }^{7}$ Deren Erforderlichkeit wird also geprüft.

Es handelt sich um zwei gesonderte, naturschutzrechtlich obligatorische Verfahrensschritte. ${ }^{8}$ In dem ersten der Vor-

1) EuGH, Urt. v. 29.7.2019 - C-411/17, ECLI:EU:C:2019:622, NuR 2019, 538, Rdnr. 119 - Inter-Environnement Wallonie (Doel). Bereits EuGH, Urt. v. 25.7.2018, C-164/17, ECLI:EU:C:2018:593, Rdnr. 32 - Grace und Sweetman.

2) EuGH, Urt. v. 29.7.2019 - C-411/17, ECLI:EU:C:2019:622, NuR 2019, 538, Rdnr. 121 - Inter-Environnement Wallonie (Doel).

3) VGH München, Urt. v. 30.9.2009 - 8 A 05.40050 u.a., NuR 2010, 355, B.II.1.2 - Westtangente Rosenheim; Frenz, in: ders./ Müggenborg, BNatSchG, 2. Aufl. 2016, $\$ 34$ Rdnr. 53 ff. auch für das Folgende; vgl. Storost, DVBl. 2009, 673, 674.

4) Beide sind zu unterscheiden, BVerwG, Urt. v. 17.1.2007 - 9 A 20.05, BVerwGE 128, 1, NuR 2007, 336, Rdnr. 40.

5) BVerwG, Urt. v. 27.11.2018 - 9 A 8/17, Rdnr. 84 - A 20 NordWest-Umfahrung Hamburg.

6) GAin Kokott, Schlussanträge v. 29.1.2004 - C-127/02, ECLI:EU:C:2004:60, NuR 2004, 788, Rdnr. 80 - Waddenvereinigung und Vogelsbeschermingvereniging.

7) BVerwG, Urt. v. 27.11.2018 - 9 A 8/17, BVerwGE 163, 380, Rdnr. 84 - A 20 Nord-West-Umfahrung Hamburg; bereits BVerwG, Urt. v. 17.1.2007 - 9 A 20.05, BVerwGE 128, 1, NuR 2007, 336, Rdnr. 40, 60; Urt. v. 14.7.2011 - 9 A 12.10, BVerwGE 140, 149, NuR 2011, 866, Rdnr. $87 \mathrm{ff}$.

8) BVerwG, Urt. v. 10.4.2013 - 4 C 3/12, BVerwGE 146, 176, NuR 2013, 656, Rdnr. 10; OVG Magdeburg, Urt. v. 26.9.2013 - 2 L 95/13, NuR 2014, 127, 128. 
prüfung, des sog. Screening, werden die Fälle ausgeschieden, in denen von vornherein durch objektive Umstände ausgeschlossen werden kann, dass sie das Gebiet einzeln oder im Zusammenwirken mit anderen Plänen oder Projekten erheblich beeinträchtigen könnten. ${ }^{9}$ Dies entspricht der Rechtsprechung des EuGH, nach der Pläne und Projekte nach Art. 6 Abs. 3 FFH-RL nur solche sind, bei denen ein solcher Ausschluss erheblicher Beeinträchtigungen nicht a priori in Betracht kommt. ${ }^{10}$

Ist damit eine erhebliche Beeinträchtigung des FFH-Gebietes offensichtlich ausgeschlossen bzw. ist sie nicht ernstlich zu besorgen, steht bereits $₫ 34$ Abs. 2 BNatSchG dem Plan bzw. dem Projekt nicht entgegen. ${ }^{11}$ Werden somit die Erhaltungsziele evident nicht beeinträchtigt, sind sie auch nicht in eine Abwägung im Rahmen einer planerischen Entscheidung oder einer ermessensgeleiteten Zulassungsentscheidung einzustellen. ${ }^{12}$

\section{Weite Konzeption des EuGH}

Damit bildet das Vorliegen einer wahrscheinlichen erheblichen Beeinträchtigung ebenso eine Vorfrage wie das Vorliegen eines Projektes. ${ }^{13}$ Diese Vorprüfung ist ein grober Filter im Hinblick darauf, ob eine detaillierte FFH-Verträglichkeitsprüfung erfolgen muss. Daher dürfen auch auf dieser Prüfungsstufe nicht vorschnell mögliche Beeinträchtigungen ausgeschieden und als unerheblich qualifiziert werden. Entsprechend weit ist auch die Wahrscheinlichkeit einer erheblichen Beeinträchtigung eines geschützten $\mathrm{Ge}-$ biets zu interpretieren. Entsprechend dem für den Habitatschutz geltenden Vorsorgeprinzip besteht eine solche $\mathrm{Ge}-$ fahr schon dann, wenn sich auf der Grundlage der besten einschlägigen wissenschaftlichen Erkenntnisse nicht ausschließen lässt, dass der Plan oder das Projekt die für dieses Gebiet festgelegten Erhaltungsziele möglicherweise beeinträchtigt; dafür zählen namentlich die besonderen Merkmale und Umweltbedingungen des von einem solchen Plan oder Projekt betroffenen Gebietes, wie der EuGH im Urteil Doel judizierte. ${ }^{14}$ Hierfür genügt die Lage in der Nähe von nach der Habitat- bzw. der Vogelschutzrichtlinie geschützten Gebieten. Die Lage eines Projektes außerhalb eines Natura 2000-Gebietes allein führt zu keiner Befreiung von den Anforderungen des Art. 6 Abs. 3 FFH-Richtlinie, ${ }^{15}$ sondern es zählen die möglichen negativen Auswirkungen auf dieses. Dementsprechend war auch der Projektbegriff namentlich für Energieanlagen weit zu ziehen. ${ }^{16}$

\section{Wahrung des günstigen Erhaltungszustands und Critical Loads}

\subsection{Erheblichkeit von Zusatzbelastungen}

Zunächst ist der fragliche Gebietsbestandteil mit dem relevanten Lebensraumtyp zu ermitteln und darauf die jeweilige Beeinträchtigung zu beziehen, bevor es um ihre Erheblichkeit geht. Dabei zählt die Belastung des fraglichen Gebietsteils insgesamt. Trifft eine Beeinträchtigung auf eine bereits vorhandene, ist mithin das Gesamtbild entscheidend. Diese Vorbelastung ist also zwingend zu berücksichtigen. ${ }^{17}$ Deshalb läuft prinzipiell jede Zusatzbelastung dem günstigen Erhaltungszustand zuwider und ist deshalb erheblich, wenn die Vorbelastung die naturschutzfachlich für das Erhaltungsziel unbedenkliche Belastungsgrenze ausschöpft oder sogar überschreitet. ${ }^{18}$ Je schlechter der Erhaltungszustand eines Gebietes ist, desto höher fallen also die Anforderungen an Zusatzbelastungen aus. Umgekehrt sind bei gutem Erhaltungszustand Bagatellschwellen denkbar. ${ }^{19}$

\subsection{Irrelevanzschwelle}

In Fällen, in denen die Vorbelastung den Critical Load um mehr als das Doppelte übersteigt, ist eine Irrelevanz- schwelle von 3\% dieses Wertes anzuerkennen. ${ }^{20}$ Der Critical Load ist der als noch verträglich anzusehende Eintrag. Diese Methode der Grenzbelastung und das Abschneidekriterium einer Zusatzbelastung von $0,3 \mathrm{~kg} / \mathrm{ha}$ a spiegelt die besten Erkenntnisse wider, um die Belastung durch Stickstoffeinträge in geschützte Lebensräume zu ermitteln. ${ }^{21}$

\subsection{Vereinbarkeit mit EuGH-Judikat vom 7. 11. 2018}

An diesem Ansatz hält das BVerwG nach dem EuGH-Urteil vom 7.11.2018 ${ }^{22}$ weiter fest. ${ }^{23}$ Auch in diesem auf ein anderes niederländisches Modell bezogenen Urteil, das auf kritische Bemerkungen der Generalanwältin ${ }^{24} \mathrm{zu}$ den deutschen Critical Loads nicht einging, ${ }^{25}$ stellte der EuGH darauf $a b$, dass aus wissenschaftlicher Sicht kein vernünftiger Zweifel bestehen darf. ${ }^{26}$ Das ist auch das Grundkonzept des BVerwG.

Allerdings hob der EuGH für das niederländische Modell darauf ab, dass die Vereinbarkeit mit Art. 6 Abs. 3 FFH-RL voraussetzt, dass sich die zuständigen Behörden Gewissheit verschafft haben, dass keines der erlaubten Projekte schädliche Auswirkungen auf das betreffende Gebiet hat. ${ }^{27}$ Dabei ist sogar eine Einzelfallgenehmigung entbehrlich. Das setzt allerdings voraus, dass sich das nationale Gericht - und damit auch die mitgliedstaatliche Zulassungsbehörde - vergewissert hat, dass die im Voraus durchgeführte angemessene Prüfung keinen vernünftigen wissenschaftlichen Zweifel daran bestehen lässt, dass diese Pläne oder Projekte keine

9) Ewer, in: Lütkes/Ewer, BNatSchG, 2. Aufl. 2018, \$34 Rdnr. 13

10) S. EuGH, Urt. v. 7.9.2004 - C-127/02, ECLI:EU:C:2004:482, NuR 2004, 788, 790 - Waddenvereniging und Vogelbeschermingsvereniging.

11) Ewer, in: Lütkes/Ewer, BNatSchG, 2. Aufl. 2018, \$34 Rdnr. 13

12) Ewer, in: Lütkes/Ewer, BNatSchG, 2. Aufl. 2018, \$34 Rdnr. 13 a.E. (allenfalls sehr geringes Gewicht) gegen VGH Kassel, Urt. v. 29.3.2012 - 4 C 694/10.N, NuR 2012, 644, 647.

13) Dazu näher Frenz, NuR 2020, Heft 1

14) EuGH, Urt. v. 29.7.2019 - C-411/17, ECLI:EU:C:2019:622, NuR 2019, 538, Rdnr. 134 - Inter-Environnement Wallonie (Doel).

15) EuGH,Urt.v. 29.7.2019-C-411/17,ECLI:EU:C:2019:622,NuR 2019, 538, Rdnr. 136 - Inter-Environnement Wallonie (Doel). Bereits EuGH, Urt. v. 10.1.2006, C-98/03, ECLI:EU:C:2006:3, NuR 2006, 166, Rdnr. 44, 51 - Kommission/Deutschland; Urt. v. 26. 4.2017, C-142/16, ECLI:EU:C:2017:301, NuR 2017, 393, Rdnr. 29 - Kommission/Deutschland.

16) Näher Frenz, NuR 2020, Heft 1.

17) BVerwG, Beschl. v. 10.11.2009 - 9 B 28.09, NuR 2010, 190, Rdnr. 3.

18) BVerwG, Urt. v. 14.4.2010 - 9 A 5.08, BVerwGE 136, 291, NuR 2010, 558, Rdnr. 91 - A 44

19) Frenz, in: ders./Müggenborg, BNatSchG, 2. Aufl. 2016, \34 Rdnr. $61 \mathrm{ff}$. auch für das Folgende.

20) BVerwG, Urt. v. 14.4.2010 - 9 A 5.08, BVerwGE 136, 291, NuR 2010, 558, Rdnr. 93 - A 44.

21) Das gilt auch für kumulative Belastungen, BVerwG, Urt. v. 15. 5. 2019 - 7 C 27/17, Rdnr. 34.

22) EuGH,Urt.v.7.11.2018-C-293u.294/17,ECLI:EU:C:2018:882, $\mathrm{NuR} 2018,852$ - Coöperatie Mobilisation for the Environment und Vereniging Leefmilieu.

23) BVerwG, Urt. v. 27.11.2018 - 9 A 8/17, BVerwGE 163, 380, Ls. 3 - A 20 Nord-West-Umfahrung Hamburg.

24) GAin Kokott, Schlussanträge vom 25.7.2018 - C-293 u. 294/17, ECLI:EU:C:2018:622, NuR 2018, 852, Rdnr. 62f. - Coöperatie Mobilisation for the Environment und Vereniging Leefmilieu.

25) BVerwG, Urt. v. 27.11.2018 - 9 A 8/17, BVerwGE 163, 380, Rdnr. 82 - A 20 Nord-West-Umfahrung Hamburg.

26) EuGH,Urt.v. 7.11.2018-C-293u.294/17,ECLI:EU:C:2018:882, NuR 2018, 852, Rdnr. 104, 112 - Coöperatie Mobilisation for the Environment und Vereniging Leefmilieu.

27) EuGH,Urt.v.7.11.2018-C-293u.294/17,ECLI:EU:C:2018:882, NuR 2018, 852, Rdnr. 104, 112 - Coöperatie Mobilisation for the Environment und Vereniging Leefmilieu. 
schädlichen Auswirkungen auf die betreffenden Gebiete als solche haben werden. ${ }^{28}$

\subsection{Keine Pauschalausnahmen}

Indes dürfen keine Pauschalausnahmen für eine bestimmte Kategorie von Projekten erfolgen; es muss zumindest anhand objektiver Umstände eine erhebliche Beeinträchtigung ausgeschlossen sein. ${ }^{29}$ Der Ansatzpunkt für die Herausnahme aus der FFH-Verträglichkeitsprüfung ist also nicht die Art des Gebietes, sondern das Unterschreiten bestimmter Werte, deren Unbedenklichkeit vorher angemessen geprüft wurde. Daraus ergibt sich dann die Unbedenklichkeit dieser Projekte. Dafür begründen die Critical Loads eine Regelvermutung. Bei ihrer Einhaltung sind vernünftige Zweifel ausgeschlossen.

Schon bisher kamen keine generalisierenden Unbedenklichkeitserklärungen ohne besondere fachliche Rechtfertigung aus. So genügte der Untersuchungsrahmen nach der TA Luft mit einer regelmäßig 50-fachen Schornsteinhöhe nicht, da er nicht auf die Bedürfnisse des Habitatschutzes ausgerichtet ist und sich daher von diesem spezifischen Maßstab entfernt. Eine Irrelevanzschwelle von $10 \%$ lag daher $\mathrm{zu}$ hoch, nicht hingegen eine solche von $3 \%$, da insoweit nach fachwissenschaftlichem Konsens keine signifikanten Änderungen eintreten. ${ }^{30}$ Es fehlt dann der in $\$ 34$ BNatSchG geforderte Zusammenhang zwischen Stickstoffeintrag eines Vorhabens und Beeinträchtigung ${ }^{31}$ und damit die Kausalität. ${ }^{32}$

\subsection{Relevanz kumulativer Belastungen auch bei späterer Antragstellung}

Die Bagatellgrenze zählt auch bei kumulativen Belastungen. Dabei sind alle genehmigten Projekte zu berücksichtigen, auch wenn sie später beantragt wurden, ohne dass stets wegen einer schleichenden Verschlechterung des Gebietszustandes durch Bagatelleinträge auf den Zeitpunkt der Unterschutzstellung zurückzugehen ist. ${ }^{33}$ Entscheidend ist nicht die Antragsabfolge, sondern die durch die vorhandenen Genehmigungen feststehende Gesamtbelastungswirkung. Letztlich beeinträchtigt sie das Schutzgebiet; die Stellung des Antrags bildet insoweit eine zurücktretende Formalie. Der Bestandsschutz ist im Habitatschutzrecht gerade in der EuGH-Judikatur generell schwach ausgeprägt. ${ }^{34}$ Es zählen eher die tatsächlichen Verhältnisse als die verfahrensmäßige Abfolge, wie auch im nächsten Punkt durch die EuGH-Entscheidung Doel deutlich wird.

\section{FFH-Verträglichkeitsprüfung und verbleibende Unsicherheiten}

\subsection{Notwendige Gewissheit als Ausschluss vernünftiger Zweifel}

Die FFH-Verträglichkeitsprüfung muss dann durchgeführt werden, sobald alle Folgen hinreichend ermittelbar sind, die das fragliche Projekt für ein geschütztes Gebiet haben kann. ${ }^{35}$ Dies ist unabhängig davon, dass ein Gesetz ergeht, auch wenn danach noch individuelle Genehmigungen für Stromerzeugung $\mathrm{zu}$ industriellen Zwecken erforderlich sind. ${ }^{36}$ Entscheidend ist, dass durch ein solches Gesetz ein solches Vorhaben ermöglicht wird.

Der EuGH bekräftigte im Urteil Doel seine strenge Grundlinie: Die Behörden dürfen ein Projekt nur genehmigen, wenn sie die Gewissheit erlangt haben, dass die Pläne oder Projekte sich nicht nachteilig auf das fragliche Gebiet auswirken. Das bedeutet, dass aus ,wissenschaftlicher Sicht kein vernünftiger Zweifel daran besteht, dass es keine solchen Auswirkungen gibt." ${ }^{37}$ Entsprechend ausführlich und abgesichert muss die Prüfung auf der Ebene der Beurteilung der FFH-Verträglichkeit ausfallen. Es bedarf einer angemessenen Prüfung eines Planes oder eines Projekts auf
Verträglichkeit für das betreffende Gebiet und damit einer Ermittlung sämtlicher Gesichtspunkte des Plans oder Projekts, die für sich oder in Verbindung mit anderen Plänen oder Projekten die für dieses Gebiet festgelegten Erhaltungsziele beeinträchtigen können. ${ }^{38}$

\subsection{Nicht ausräumbare wissenschaftliche Unsicherheiten}

Dabei können zwar Prognosen und Risikoabschätzungen getroffen werden. Besonders problematisch ist, wenn nicht ausräumbare wissenschaftliche Unsicherheiten bestehen. Der EuGH verlangt zwar eine Gewissheit, dass sich eine zu genehmigende Tätigkeit nicht nachteilig auf das FFH-Gebiet als solches auswirkt. Das ist allerdings schon ,dann der Fall, wenn aus wissenschaftlicher Sicht kein vernünftiger Zweifel daran besteht, dass es keine solchen Auswirkungen gibt.". ${ }^{39}$ Dementsprechend liegt eine solche Gewissheit nach dem BVerw ${ }^{40}$ auch dann vor, wenn die durch Analogieschlüsse, Wahrunterstellungen und Worst-Case-Annahmen erzielbaren Ergebnisse für die untersuchten Fragestellungen ,,auf der sicheren Seite“ liegen. ${ }^{41}$ Sie müssen zudem den untersuchten Sachverhalt sachdienlich erfassen sowie ein differenziertes Schutzkonzept ermöglichen. ${ }^{42}$

\subsection{Möglichkeit eines Monitoring}

Es genügt ein wirksames Risikomanagement mit hinreichendem Monitoring, wie das BVerwG-Urteil zur Elbver-

28) EuGH, Urt. v. 7.11.2018 - C-293 u. 294/17 u.a., ECLI:EU:C 2018:882, NuR 2018, 852, Ls. 4, Rdnr. 104 - Coöperatie Mobilisation for the Environment und Vereniging Leefmilieu.

29) EuGH, Urt. v. 7.11.2018 - C-293 u. 294/17 u.a., ECLI:EU:C: 2018:882, Ls. 5, Rdnr. 112 - Coöperatie Mobilisation for the Environment und Vereniging Leefmilieu.

30) BVerwG, Urt. v. 14.4.2010 - 9 A 5.08, BVerwGE 136, 291, NuR 2010, 558, Rdnr. 92 - A 44.

31) BVerwG, Urt. v. 23.4.2014 - 9 A 25.12, BVerwGE 149, 289 , NuR 2014, 706, Rdnr. 45 - A 49; BVerwG, Urt. v. 27.11.2018 - 9 A 8.17, BVerwGE 163, 380, Rdnr. 80.

32) Balla u.a., Waldökologie, Landschaftsforschung und Naturschutz, Dezember 2014, $43 \mathrm{ff}$.

33) BVerwG, Urt. v. 15.5.2019 - 7 C 27/17, Rdnr. 45 ff. in Aufhebung des Urteils des OVG Münster v. 26.6.2016 - 8 D 99/13 AK.

34) Näher Frenz, NuR 2020, Heft 1.

35) EuGH, Urt. v. 29.7.2019 - C-411/17, ECLI:EU:C:2019:622, NuR 2019, 538, Rdnr. 143 - Inter-Environnement Wallonie (Doel).

36) EuGH, Urt. v. 29.7.2019 - C-411/17, ECLI:EU:C:2019:622, NuR 2019, 538, Rdnr. 144f. - Inter-Environnement Wallonie (Doel).

37) EuGH, Urt. v. 29.7.2019 - C-411/17, ECLI:EU:C:2019:622, NuR 2019, 538, Rdnr. 119f. - Inter-Environnement Wallonie (Doel); m.w. N. EuGH, Urt. v. 7.11.2018- C-461/17, ECLI:EU:C:2018:883, NuR 2018, 848, Rdnr. 33 - Holohan u. a.; auch BVerwG, Urt. v. 17.1.2007 - 9 A 20.05, BVerwGE 128, 1, NuR 2007, 336, Rdnr. 41 - Westumfahrung Halle.

38) EuGH, Urt. v. 29.7.2019 - C-411/17, ECLI:EU:C:2019:622, NuR 2019, 538, Rdnr. 120 - Inter-Environnement Wallonie (Doel).

39) EuGH, Urt.v. 29. 7. 2019-C-411/17,ECLI:EU:C:2019:622, NuR 2019, 538, Rdnr. 120 - Inter-Environnement Wallonie (Doel); bereits Urt. v. 7.11.2018 - C-461/17, ECLI:EU:C:2018:883, Rdnr. 33 - Holohan u. a.m. w. N.

40) Anders wohl Möckel, in: Schlacke (Hrsg.), GK-BNatSchG, 2. Aufl. 2016, $\$ 34$ Rdnr. 70.

41) BVerwG, Urt. v. 17.1.2007 - 9 A 20.05, BVerwGE 128, 1, NuR 2007, 336, Rdnr. 64 - Westumfahrung Halle.

42) BVerwG, Urt. v. 17.1.2007 - 9 A 20.05, BVerwGE 128, 1, NuR 2007, 336, Rdnr. 64 - Westumfahrung Halle; Urt. v. 6.11.2013 - 9 A 14.12, BVerwGE 148, 373, NuR 2014, 262, Rdnr. 51; Urt. v. 14.7.2011 - 9 A 12.10, BVerwGE 140, 149, NuR 2011, 866, Rdnr. 71. 
tiefung bestätigte. ${ }^{43}$ Damit verbleiben zwar Ungewissheiten. Sie gehen aber nicht über vernünftige Zweifel hinaus, welche nicht mehr vorliegen dürfen. Vielmehr besteht auch beim Monitoring eine flankierende Sicherung. Durch die Anordnung von Beobachtungsmaßnahmen können gerade bei wissenschaftlicher Unsicherheit über die Wirksamkeit von Schutzmaßnahmen, welche das Vorliegen einer erheblichen Beeinträchtigung i.S.v. $\$ 34$ Abs. 2 bzw. Art. 6 Abs. 3 FFH-RL hindern, weitere Erkenntnisse über die Beeinträchtigungen gewonnen werden, um die Durchführung des Vorhabens zu steuern. ${ }^{44}$

Diese Wirksamkeit kann zwar nicht allein durch das Monitoring belegt werden. Allerdings wird das Monitoring als notwendiger Bestandteil des Risikomanagements gesehen, das die fortdauernde ökologische Funktion der Schutzmaßnahmen gewährleistet. Mit ihm müssen Korrektur- und Vorsorgemaßnahmen, welche die Risiken für Erhaltungsziele des betroffenen Schutzgebiets wirksam ausräumen, für den Fall einhergehen, dass sich bei der Beobachtung die positive Prognose der Wirksamkeit von Schutzmaßnahmen als falsch erweist. ${ }^{45}$ Damit besteht die Möglichkeit nachträglicher Korrektur.

\subsection{Keine verbleibenden Zweifel}

Ein „Nullrisiko“, eine völlige Gewissheit ${ }^{46}$ zum Zeitpunkt der Entscheidung ist hingegen nicht verlangt. Das gilt auch und gerade nach der Rechtsprechung des EuGH. Dieser verlangt nur die Ausschöpfung aller wissenschaftlichen Mittel und Quellen ${ }^{47}$ und prüft auf dieser Basis, ob vernünftige $Z$ weifel verbleiben, dass erhebliche Beeinträchtigungen vermieden werden. ${ }^{48}$ Auch das BVerwG bezieht sich, wenn auch nur besonders herausgehoben, auf den Fall wissenschaftlicher Erkenntnisse. Es ist allerdings Ausdruck der Beweislastverteilung, dass sämtliche Risiken aus Schwierigkeiten bei der Umsetzung oder aus der langfristigen Beurteilung von solchen eine erhebliche Beeinträchtigung hindernden Maßnahmen zulasten des Vorhabens gehen, wie das BVerwG zurecht betonte. ${ }^{49}$ Die Behörde muss dabei eine langfristige Effektivität gewährleisten. ${ }^{50}$

Um mit dem Doel-Urteil zu schließen: Zunächst sind sämtliche Gesichtspunkte des Plans oder Projekts zu ermitteln, die für sich oder in Verbindung mit anderen Plänen oder Projekten die für dieses Gebiet festgelegten Erhaltungsziele beeinträchtigen können. Sodann muss die Gewissheit in Form des Fehlens vernünftiger Zweifel erlangt werden, dass es keine solchen Auswirkungen gibt. ${ }^{51}$ Verbleiben Zweifel, kann das Vorhaben nicht genehmigt werden.

\section{Keine Ersetzung durch die Prüfung von Mindeststandards}

\subsection{Notwendige spezifische Prüfung, keine bloße Vorprüfung}

Die FFH-Verträglichkeitsprüfung muss als solche durchgeführt werden. Es bedarf einer spezifischen Prüfung nach dem System von Art. 6 Abs. 3 und Abs. 4 FFHRL. Sie kann daher nicht dadurch ersetzt werden, dass vor der Aufnahme eines Gebietes in die Gemeinschaftsliste ein zu diesem Zeitpunkt zwar genehmigtes, aber noch nicht ausgeführtes Projekt eine Einhaltung der Mindeststandards entsprechend den mitgliedstaatlichen Schutzpflichten nach Art. 6 Abs. 2 FFH-RL ${ }^{52}$ geprüft wurde. ${ }^{53}$ Das gilt auch dann, wenn eine FFH-Verträglichkeitsprüfung nachträglich erforderlich wird, weil das Gebiet erst dann in die Liste der Gebiete von gemeinschaftlicher Bedeutung aufgenommen wurde und sich daher aus Art. 6 Abs. 2 FFH-RL eine mitgliedstaatliche Schutzpflicht ergibt, die geeigneten Maßnahmen zu ergreifen: Können die Schutzziele des Gebietes nur über eine nachträgli- che Verträglichkeitsprüfung erreicht werden, konkretisiert sich die allgemeine Schutzpflicht in eine Pflicht zur Durchführung dieser Prüfung. ${ }^{54}$ Dahin wird sie damit verdichtet und fokussiert. Dabei sind, um ein einheitliches Schutzniveau zu gewährleisten, die Standards nach Art. 6 Abs. 3 FFH-RL zugrunde zu legen; diese Vorschrift ist mit Art. 6 Abs. 2 FFH-RL als einheitlicher Normkomplex zu sehen. ${ }^{55}$

\subsection{Ausdruck des Effektivitätsgrundsatzes}

Eine bloße Vorprüfung oder Gefährdungsabschätzung genügen nicht. Der unionsrechtliche Effektivitätsgrundsatz verlangt eine Zugrundelegung der aktuellen Situation und nicht die unter Umständen Jahre zurückliegende zum Genehmigungszeitpunkt. ${ }^{56}$ Vielmehr müssen alle bei der Aufnahme eines Projekts in die Liste der Gebiete von gemeinschaftlicher Bedeutung vorliegenden Umstände und alle danach durch die teilweise oder vollständige Ausführung dieses Plans oder Projekts eingetretenen oder möglicherweise eingetretenen Auswirkungen berücksichtigt werden. ${ }^{57}$ Schließlich war das Gebiet vorher noch gar nicht

43) BVerwG, Urt. v. 9.2.2017 - 7 A 2/15, BVerwGE 158, 1, NuR 2017, 552, Rdnr. 226 - Elbvertiefung; BVerwG, Urt. v. 17. 1. 2007 - 9 A 20.05, BVerwGE 128, 1, NuR 2007, 336, Rdnr. 55, 64, 66 - Westumfahrung Halle; BVerwG, Urt. v. 6.11.2013 - 9 A 14.12, NuR 2014, 277, Rdnr. 56; BVerwG, Urt. v. 28.3. 2013 - 9 A 22.11, BVerwGE 146, 145, NuR 2013, 565, Rdnr. 95; BVerwG, Urt. v. 12.3.2008 - 9 A 3.06, BVerwGE 130, 299, NuR 2008, 633, Rdnr. 105

44) BVerwG, Urt. v. 17.1.2007 - 9 A 20.05, BVerwGE 128, 1, NuR 2007, 336, Rdnr. 55 - Westumfahrung Halle unter Verweis auf GAin Kokott, Schlussanträge v. 29.1.2004 - C-127/02, ECLI:EU:C:2004:60, Rdnr. 108 - Waddenvereniging und Vogelbeschermingsvereniging.

45) BVerwG, Urt. v. 9.2.2017 - 7 A 2/15, BVerwGE 158, 1, NuR 2017, 552, Rdnr. 226 - Elbvertiefung; BVerwG, Urt. v. 17.1.2007 - 9 A 20.05, BVerwGE 128, 1, NuR 2007, 336, Rdnr. 55 - Westumfahrung Halle, auch noch für Kompensationsmaßnahmen, die aber nach dem EuGH-Urteil Briels (v. 15.5.2014 - C-521/12, ECLI:EU:C:2014:330, NuR 2014, 487) insoweit ausgeschlossen sind.

46) So Gellermann, in: Landmann/Rohmer, UmweltR, Stand: 2/2019, \34 BNatSchG Rdnr. 26; im Anschluss an BVerwG, Urt. v. 17.1.2007 - 9 A 20/05, BVerwGE 128, 1, NuR 2007, 336, Rdnr. 54 und gegen Jarass, NuR 2007, 371, 374: hinreichende Wahrscheinlichkeit negativer Wirkungen.

47) Siehe GAin Kokott, Schlussanträge v. 29.1.2004 - C-127/02, ECLI:EU:C:2004:60, Rdnr. 97.

48) EuGH, Urt. v. 26.10.2006 - C-239/04, ECLI:EU:C:2006:665, Rdnr. 20 - Kommission/Portugal.

49) BVerwG, Urt. v. 9.2.2017 - 7 A 2/15, BVerwGE 158, 1, NuR 2017, 552, Rdnr. 226 - Elbvertiefung; BVerwG, Urt. v. 17. 1. 2007 - 9 A 20.05, BVerwGE 128, 1, NuR 2007, 336, Rdnr. 54 Westumfahrung Halle, jeweils unter Verweis auf EuGH, Urt. 29.1.2004 - C-209/02, ECLI:EU:C:2004:61, NuR 2004, 656, Rdnr. 24 ff. - Kommission/Österreich.

50) Frenz, in: ders./Müggenborg, BNatSchG, 2. Aufl. 2016, \$34 Rdnr. 104 auch zum Vorhergehenden.

51) EuGH, Urt. v. 29.7.2019 - C-411/17, ECLI:EU:C:2019:622, $\mathrm{NuR}$ 2019, 538, Rdnr. 119f. - Inter-Environnement Wallonie (Doel).

52) Siehe EuGH, Urt. v. 13. 1.2005 - C-117/03, ECLI:EU:C:2005:16, NuR 2005, 242 - Dragaggi.

53) Siehe die EuGH-Vorlage des BVerwG, Beschl. v. 6.3.2014-9 C 6.12, NuR 2014, 633, Rdnr. 35

54) EuGH, Urt. v. 14.1.2016 - C-399/14, ECLI:EU:C:2016:10, NuR 2016, 119, Rdnr. 37, 44 - Grüne Liga Sachsen u.a.

55) EuGH, Urt. v. 14.1.2016 - C-399/14, ECLI:EU:C:2016:10, NuR 2016, 119, Rdnr. 52 ff. - Grüne Liga Sachsen u. a.

56) Bereits EuGH-Vorlage des BVerwG, Beschl. v. 6.3.2014 - 9 C 6.12, NuR 2014, 633, Rdnr. 39

57) EuGH, Urt. v. 14.1.2016 - C-399/14, ECLI:EU:C:2016:10, NuR 2016, 119, Rdnr. 60 ff. - Grüne Liga Sachsen u. a. 
dem Habitatschutz unterstellt (Art. 4 Abs. 5 FFH-RL). ${ }^{58}$ Dies entschied der EuGH für die Waldschlösschenbrücke in Dresden und im Anschluss daran das BVerwG: Die Verträglichkeitsprüfung ist auf den aktuellen Zeitpunkt zu beziehen und nicht auf den vor der Gebietslistung. ${ }^{59}$

\subsection{Zurücktreten der Bestandskraft}

Damit bleibt indes die Bestandskraft von Genehmigungen praktisch unberücksichtigt. ${ }^{60}$ Generell betonte der EuGH die Bedeutung rechtskräftiger Entscheidungen, die nicht mehr in Frage gestellt werden müssen. ${ }^{61}$ Indes verstößt die gänzliche Außerachtlassung von EU-Vorschriften und ihrer Durchsetzung gegen den Effektivitätsgrundsatz. Insoweit besteht eine angemessene Rechtfertigung auch nicht durch den Grundsatz der Rechtssicherheit. ${ }^{62}$ Damit können vor allem nicht nationale Verfahrensbesonderheiten die Durchsetzung von EU-Recht hindern. Ansonsten hätten es die Mitgliedstaaten in der Hand, trotz dessen Vorrangs die Anwendung von EU-Recht zu umgehen.

Das BVerwG hob in seinem Vorlagebeschluss an den EuGH darauf ab, ob bereits eine gerichtliche Prüfung erfolgte - und sei es in einem vorläufigen Rechtschutzverfahren. ${ }^{63}$ Dann soll sogar unschädlich sein, wenn sich die tatsächliche Situation zur Zeit der Genehmigungserteilung nicht mehr (voll) rekonstruieren lässt: Unterlagen und Untersuchungen vor Baubeginn reichen aus, Ungewissheiten dürfen nicht zulasten des Vorhabenträgers gehen. ${ }^{64}$ Dies lässt allerdings den gerade im Habitatschutz so sehr vom EuGH betonten Effektivitätsgrundsatz in den Hintergrund treten.

\subsection{Unbeachtlichkeit vorläufigen Rechtsschutzes}

Der EuGH hält daher die Anforderungen in vollem Umfang aufrecht. Sie können nicht deshalb verändert werden, weil die Entscheidung zur Genehmigung dieses Plans oder Projekts sofort vollziehbar und ein Verfahren des vorläufigen Rechtsschutzes unanfechtbar erfolglos geblieben war. ${ }^{65}$

\subsection{Alternativenprüfung}

Damit kann ein Projekt möglicherweise sogar wieder abgerissen werden müssen. Insoweit ist eine Alternativenprüfung mit einer Begrenzung der Nutzung und einer Schließung des fraglichen Bauwerks vorzunehmen; insoweit sind die jeweiligen ökologischen Folgen mit den überwiegenden öffentlichen Interessen abzuwägen, die zur Errichtung des Bauwerks geführt haben. Das erfolgt im Rahmen der eng auszulegenden Ausnahmevorschrift des Art. 6 Abs. 4 FFH-RL; dabei kann nicht nur auf die wirtschaftlichen Kosten solcher Maßnahmen abgestellt werden. ${ }^{66}$ Auch insoweit kann der Maßstab nicht deshalb verändert werden, weil der Plan oder das Projekt bereits ausgeführt worden ist.

\section{Fazit}

Die jüngste Rechtsprechung betont auch für die Prüfungsstufe der erheblichen Beeinträchtigungen und der Ver- träglichkeit mit den Erhaltungszielen des beeinträchtigten Gebietes die notwendige Wirkungsbezogenheit des Habitatschutzes. Es dürfen für die Zulassung eines Vorhabens keine vernünftigen Zweifel negativer Auswirkungen verbleiben, wie der EuGH wieder im Doel-Urteil betonte. Damit ist zwar eine Beweislastumkehr verbunden, jedoch keine völlige Gewissheit verlangt und ein Monitoring entsprechend dem BVerwG im Elbvertiefungs-Urteil weiterhin möglich, allerdings mit der Maßgabe, dass eine langfristige Beobachtung erfolgen muss. Die FFHVerträglichkeitsprüfung verträgt keine Herabstufung zu Mindeststandards, auch wenn sie erst nachträglich erforderlich wird. Der Bestandsschutz tritt auch insoweit hinter die Effektivität des Habitatschutzes zurück. Gleichwohl können Beeinträchtigungen von vornherein ausgeschieden werden, denen die Erheblichkeit sicher fehlt. Daher sind die Critical Loads weiterhin heranziehbar, auch nach dem EuGH-Urteil vom 7.11.2018 zu einem niederländischen Modell. Es dürfen nur keine Pauschalausnahmen erfolgen. Zudem sind alle Einträge aus genehmigten Projekten zu berücksichtigen, unabhängig von der Reihenfolge der Antragstellung, wie das BVerwG zurecht entgegen dem OVG Münster entschied.

Open Access. Dieser Artikel wird unter der Creative Commons Namensnennung 4.0 International Lizenz veröffentlicht, welche die Nutzung, Vervielfältigung, Bearbeitung, Verbreitung und Wiedergabe in jeglichem Medium und Format erlaubt, sofern Sie den/die ursprünglichen Autor(en) und die Quelle ordnungsgemäß nennen, einen Link zur Creative Commons Lizenz beifügen und angeben, ob Änderungen vorgenommen wurden.

Die in diesem Artikel enthaltenen Bilder und sonstiges Drittmaterial unterliegen ebenfalls der genannten Creative Commons Lizenz, sofern sich aus der Abbildungslegende nichts anderes ergibt. Sofern das betreffende Material nicht unter der genannten Creative Commons Lizenz steht und die betreffende Handlung nicht nach gesetzlichen Vorschriften erlaubt ist, ist für die oben aufgeführten Weiterverwendungen des Materials die Einwilligung des jeweiligen Rechteinhabers einzuholen.

Weitere Details zur Lizenz entnehmen Sie bitte der Lizenzinformation auf http://creativecommons.org/licenses/by/4.0/deed.de.

Open access funding provided by Projekt DEAL.

58) EuGH, Urt. v. 14.1.2016 - C-399/14, ECLI:EU:C:2016:10, NuR 2016, 119, Rdnr. 58f. - Grüne Liga Sachsen u.a.

59) BVerwG, Urt. v. 15.7.2016 - 9 C 3.16, Rdnr. 42; näher zum Ganzen Müller-Mitschke, NuR 2018, 453, 456.

60) S. bereits Frenz, NuR 2020, Heft 1.

61) EuGH, Urt. v. 16.3.2006 - C-234/04, ECLI:EU:C:2006:178, Slg 2006, I-2585, Rdnr. 20 f. - Kapferer.

62) EuGH, Urt. v. 11.11.2015 - C-515/14, ECLI:EU:C:2016:3, Rdnr. 45f. - Klausner-Holz für das Beihilfenverbot.

63) EuGH-Vorlage des BVerwG, Beschl. v. 6.3.2014 - 9 C 6.12, NuR 2014, 633, Rdnr. 45.

64) EuGH-Vorlage des BVerwG, Beschl. v. 6.3.2014 - 9 C 6.12, NuR 2014, 633, Rdnr. 45.

65) EuGH, Urt. v. 14.1.2016 - C-399/14, ECLI:EU:C:2016:10, NuR 2016, 119, Rdnr. 68 - Grüne Liga Sachsen u. a.

66) EuGH, Urt. v. 14.1.2016 - C-399/14, ECLI:EU:C:2016:10, NuR 2016, 119, Rdnr. 73 ff. - Grüne Liga Sachsen u. a. 